\title{
Statistics of Binary and Multiple Young Stars Observed by Lunar Occultations
}

\author{
Andrea Richichi \\ European Southern Observatory, Karl-Schwarzschildstr. 2, D-85748 \\ Garching b.M. - Germany
}

\begin{abstract}
The application of the lunar occultation method to studies of young stars is discussed. The statistics of results on binarity and multiplicity is reviewed.
\end{abstract}

\section{Introduction}

Lunar occultations (LO) have provided a major contribution to the study of the binary frequency among young stars. In spite of the fact that the Moon covers only about $\approx 10 \%$ of the sky in its 18.5 years cycle, its orbit happens to go through two of the nearest, richest and best surveyed star-forming regions (SFRs). In particular, it spans continuously a large fraction of the TaurusAuriga SFR, and it goes over the $\rho$ Oph core of the Scorpius-Ophiuchus region in short episodes approximately every six years.

Simon and collaborators (1987) were the first to realize the potential offered by this lucky combination. Although LO suffer from several obvious drawbacks, they are a simple method to achieve very high angular resolution coupled with good sensitivity. In fact, to this day they still offer a better performance than speckle interferometry (SI) for what concerns resolution, and long baseline interferometry (LBI) for what concerns sensitivity.

With the introduction of LBI at very large telescopes such as the Keck and the VLTI, this special niche occupied by LO will probably cease to exist. It is then perhaps appropriate to draw now a summary of what LO have achieved in this area.

I will first provide a basic review of the LO method, and its performance with special consideration to its application for the study of young binaries. I will subsequently provide a census of binary and multiple systems among young stars, observed so far by this method.

\section{Lunar Occultations for Studies of Young Binary Stars}

In a lunar occultation event, the limb of the Moon acts as a diffracting edge. As it moves over the occulted source, a characteristic light curve is produced. Given the typical speed of the lunar motion $\left(0.5^{\prime \prime} / \mathrm{sec}\right)$, LO must be recorded with high temporal resolution. Sampling rates between 100 and $1000 \mathrm{~Hz}$ are usually employed. For the subject of interest here, i.e. binary or multiple stars, two or more diffraction patterns are observed. 


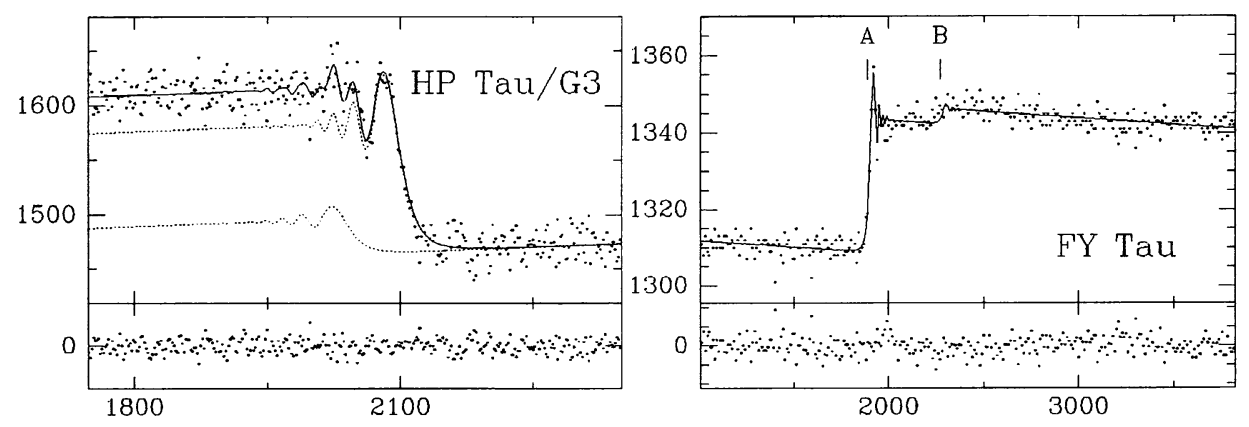

Figure 1. Two examples of actual LO light curves of young binaries. In all plots, the dots are the data points, and the solid lines the best fits. The bottom panels are the fit residuals. Ordinate axes are detector counts, abscissae axes are relative time in ms. In the case on the left, the diffraction patterns of the two components are also shown independently for clarity. The figures are adapted from Richichi et al. (1994).

In a practical observation, an important factor is the large background, caused by the light from the bright limb scattered through the atmosphere. The magnitude of this effect has been studied from a statistical database of several hundreds of observations, and it has been shown how it can be drastically reduced by the use of panoramic detectors (Richichi 1994, Richichi et al. 1996). Moreover, the companion can be substantially fainter than the primary, and also so close that the two diffraction patterns are effectively superimposed. These aspects are illustrated by two examples of actual observations, shown in Fig. 1.

The actual ability to detect a binary companion by the LO method is a function of several parameters. The most important ones are the SNR with which the lightcurve is recorded, the brightness ratio and the separation. Richichi et al. (1996) have presented a study of the detection rate, based on a large number of simulations. As a rule of thumb, the LO method can be considered sensitive to binary separations above 5 mas, but more effective for separations of about 20 mas or more. The magnitude limit of an InSb photometer at a $4 \mathrm{~m}$ class telescope is about $\mathrm{K} \lesssim 10$, and 1-2 mags can be gained by using an array detector (Richichi 1994, Richichi et al. 1996). Detectable brightness ratios can range from $\approx 1$ to 4 mags.

\section{Database and Statistics}

Fig. 2 summarizes the history of LO observations of young stars, and the statistics of observed binary and multiple systems. The bibliographical basis for this compilation is not reported in full here for reasons of space. However, it can be noted that all observational work in this field can be traced to the efforts of two groups, one led by M. Simon and the other by C. Leinert. The latest publications of these groups are are Simon et al. (1999) and Richichi et al. (1999) respectively. References to previous papers can be found therein. 


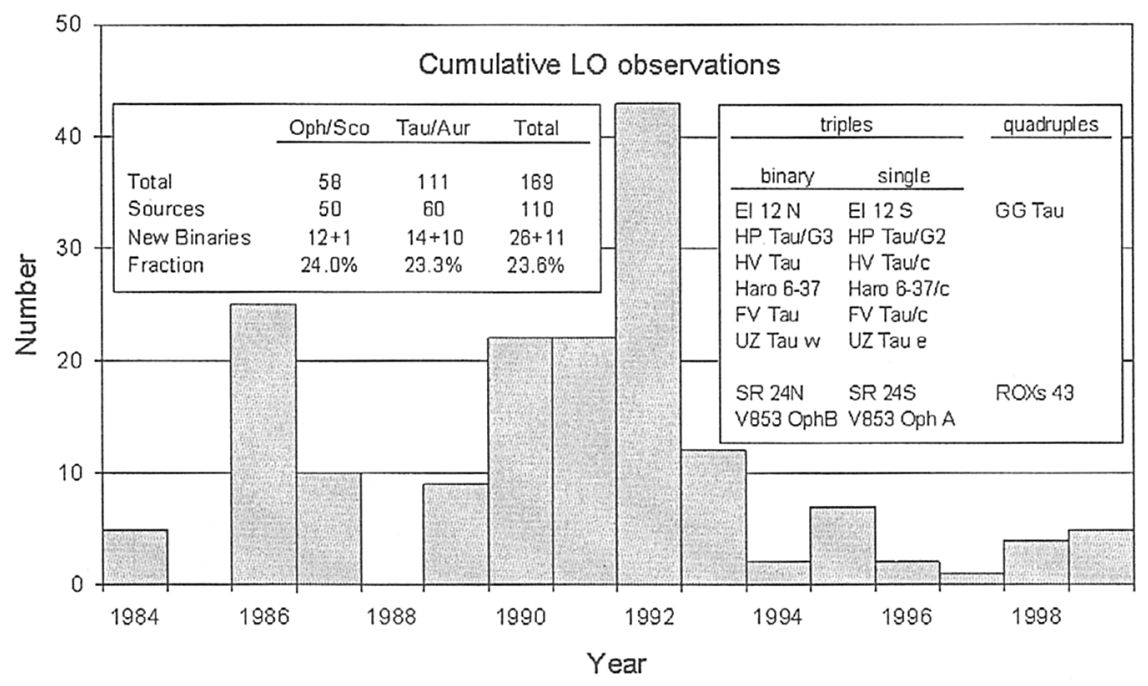

Figure 2. Statistics of the LO observations of young stars, and of the binary and multiple systems discovered by this method. Seven yet unpublished observations are included. See text for details.

A few comments on the data shown in Fig. 2 are in order. It can be seen how the peak of observations was in 1992, and the one before in 1986: this corresponds to the occurrence of passages of the Moon over the $\rho$ Oph SFR, in addition to the regular passages over the Tau SFR. Since 1992, the number of observations recorded has diminished, due to a combination of the fact that the Moon has scanned the less rich southern regions of Tau, and of less fortunate weather conditions.

For what concerns the statistics of binary and multiple systems, I have included only those systems for which at least one component has been discovered by LO. Naturally, several of these systems have been later studied also by other methods such as speckle and adaptive optics. Similarly, LO have detected binary components in many systems, which were already known by other methods (mostly speckle interferometry): these are not included in the present statistics.

\section{Concluding Remarks}

It can be stated that the binary statistics summarized in Fig. 2 are not going to change significantly in the future. One reason is that a first cycle of the orbit of the Moon over the Tau-Aur SFR is almost completed since the first observations of 1984. In this period, 3 passages over the compact $\rho$ Oph region have also occurred. Therefore, discoveries of new companions are less likely.

Another, perhaps more conclusive reason, is that in a short time we should begin to see the first large interferometric facilities such as the Keck and the 
VLTI in operation. The expected performances should equal or surpass the sensitivity and resolution of LO, while offering at the same time the possibility to choose targets and times of observation without limitations. If these predictions are respected, one can then assume, that the interest in LO will gradually fade.

There is however one last point that should be stressed, and that could make LO results still quite valuable, from a different point of view. So far, the emphasis has been on the detection of binaries. This was the novel aspect about one decade ago. The evidence for a high fraction of binaries in (at least some) SFRs, has prompted a revision of star-formation mechanisms. It has been sometimes claimed that the data for Taurus are consistent with all stars forming as members of binary or multiple systems.

Table 1. Repeated observations

\begin{tabular}{crrrrrr}
\hline Repeats & \multicolumn{2}{c}{ All } & & \multicolumn{3}{c}{ Singles } \\
\cline { 2 - 3 } \cline { 5 - 7 } & Oph & Tau & & Oph & Tau & Total \\
\hline 2 & 8 & 29 & & 5 & 22 & 27 \\
3 & 0 & 13 & & 0 & 10 & 10 \\
4 & 0 & 6 & & 0 & 4 & 4 \\
5 & 0 & 1 & & 0 & 1 & 1 \\
6 & 0 & 1 & & 0 & 1 & 1 \\
\hline \hline
\end{tabular}

In Table 1, I have summarized the frequency of multiple observations of a same young star by LO, for the whole sample and separately for stars found to be without companions. It can be seen that in a signficant number of cases, no companions were detected even in multiple LO observations. This lends statistical significance to the result. For instance, 16 stars in Taurus have been observed as single 3 or more times by LO. The record-holder is IQ Tau (6 times).

This evidence can be used to constrain theoretical predictions of how many sources are indeed produced as single stars by the star-formation mechanisms.

\section{References}

Richichi, A. 1994, IAU Symp. 158, ed. W. J. Tango, J. G. Robertson, 71

Richichi, A., Leinert, Ch., Jameson, R., Zinnecker, H. 1994, A\&A, 287, 145

Richichi, A., Baffa, C., Calamai, G., Lisi, F. 1996, AJ, 112, 2786

Richichi, A., Köhler R., Woitas J., Leinert, Ch. 1999, A\&A, 346, 501

Simon, M., Howell, R. R., Longmore, A. J., Wilking, B. A., Peterson, D. M., Chen, W.-P. 1987, ApJ, 320, 344

Simon, M., Beck, T. L., Greene, T. P., Howell, R. R., Lumsden, S., Prato, L. 1999, AJ, 117, 1594 

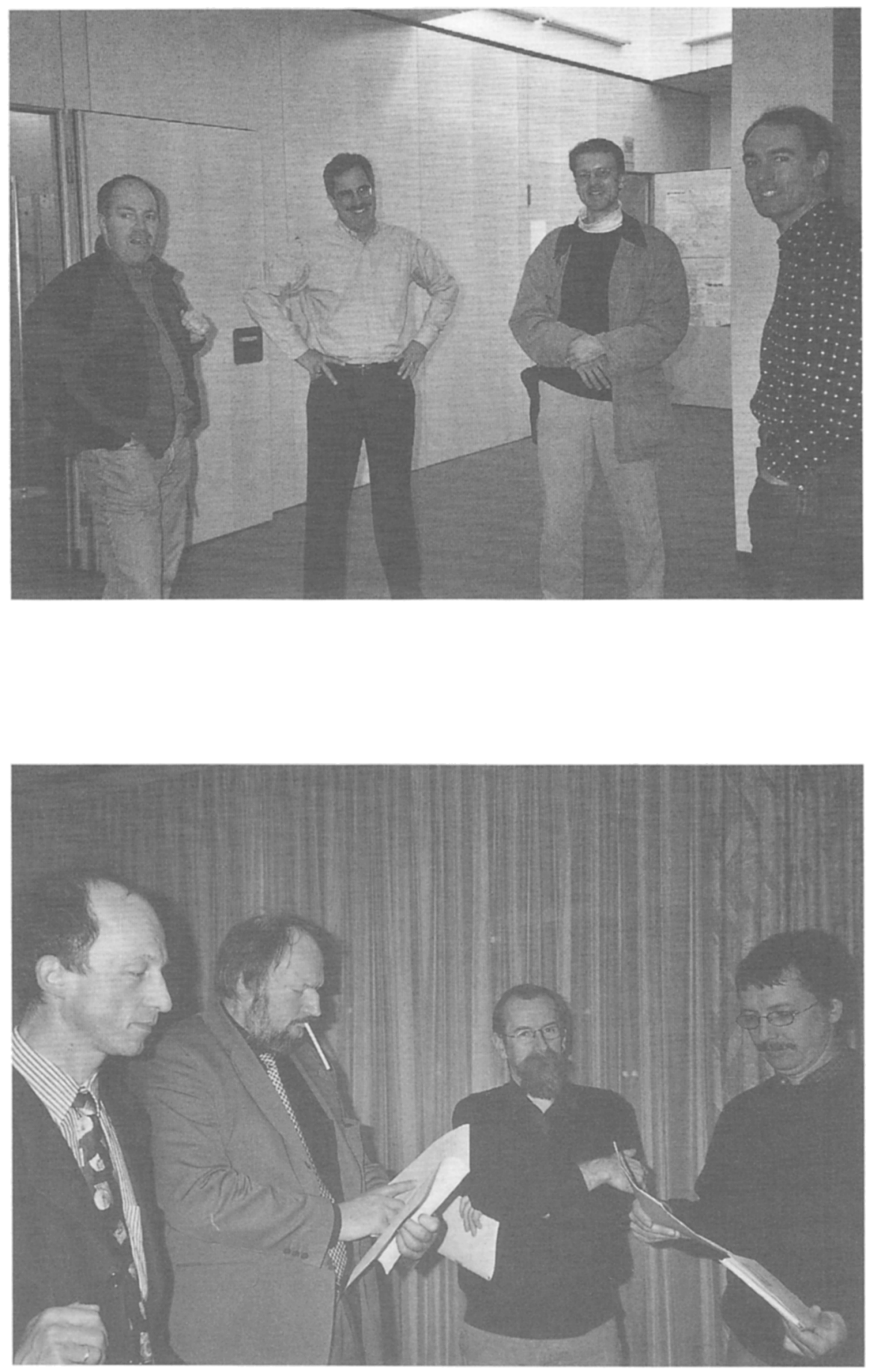

Two Trapezium-type quadruple systems:

McCaughrean, Mathieu, Jensen, Bate (top, from left)

Henning, Stecklum, Rüdiger, Wambsganß (bottom, from right) 